\title{
Toward the development of a Three-Dimensional Mid-Field Microscope
}

\author{
Yuval Garini ${ }^{* a}$, Vladimir G. Kutchoukov ${ }^{\mathrm{b}}$, Andre Bossche ${ }^{\mathrm{b}}$, Paul F.A. Alkemade ${ }^{\mathrm{c}}$, Margreet W. \\ Docter $^{\mathrm{a}}$, Piet W. Verbeek ${ }^{\mathrm{a}}$, Lucas J. van Vliet ${ }^{\mathrm{a}}$, and I.T. Young ${ }^{\mathrm{a}}$ \\ ${ }^{a}$ Imaging Science and Technology, ${ }^{\mathrm{b}}$ Micro-Electronics and ${ }^{\mathrm{c}}$ Nanoscience departments \\ Delft University of Technology, Faculty of Applied Sciences, Lorentzweg 1, Delft 2628 CJ, The \\ Netherlands \\ * Y.Garini@ tnw.tudelft.nl; Tel: +31-15-278-8574, fax: +31-15-278-6740, www.ph.tn.tudelft.nl/ yuval
}

\begin{abstract}
Recently, an extraordinary transmission of light through small holes $(<200 \mathrm{~nm})$ in a thin metallic film has been described. This phenomenon has been shown to be the result of the photon-plasmon interaction in thin films where a periodic structure (such as a set of holes) is embedded in the film. One of the extraordinary results is that the beam that passes through a hole has a very small diffraction in extreme contrast to the wide angle predicted by diffraction theory.

Based on this effect, we propose here a new type of microscopy that we term mid-field microscopy. It combines an illumination of the sample through a metallic hole-array with far-field collection optics, a scanning mechanism and a CCD. When compared to other high resolution methods, what we suggest here is relatively simple because it is based on a thin metallic film with an array of nano-sized holes. Such a method can be widely used in high-resolution microscopy and provide a novel simple-to-use tool in many life-sciences laboratories.

When compared to near-field scanning optical microscopy (NSOM), the suggested mid-field method provides a significant improvement. This is chiefly for three reasons: 1 . The penetration depth of the microscope increases from a few nanometers to a few micrometers, hence the name mid-field microscope. 2. It allows one to measure an image faster because the image is measured through many holes in parallel rather then through a single fiber tip used in conventional near-field microscopy, and 3. It enables one to perform three-dimensional reconstruction of images due to a semi-confocal effect.

We describe the physical basics of the photon-plasmon interaction that allows the coupling of light to the surface plasmons and determines the main spectral characteristics of the device. This mechanism can be ascribed due to the super-periodicity of the electron oscillations on the metallic surface engendered by the grating-like structure of the holearray.
\end{abstract}

Keywords: High resolution, near field, surface plasmons, optical microscopy

\section{INTRODUCTION}

Optical microscopy is a widely used method all across sciences. It is based on the physical principles of light and optics and provides a broad set of tools that allows one to explore small objects with high magnification and sensitivity in a relatively simple and fast manner. Nevertheless, optical microscopy has its limitations. One of the more critical limitations is the diffraction limit of light discovered first by $\mathrm{Abbe}^{1}$ in 1873 and later on fully explored and understood ${ }^{2}$.

The diffraction limit of light sets a limit to the smallest object size that can be observed, or to the smallest separation that can be distinguished between two different objects. This limit is approximately half of the wavelength of the light being used.

Various optical microscopy methods are continuously being developed to overcome the diffraction limit of light. Two of the more relevant methods include confocal microscopy and near field microscopy. Confocal microscopy only 
slightly improves the spatial resolution of conventional microscopy ${ }^{3}$ (a factor of $\sqrt{2}$ ). Near field microscopy ${ }^{4}$ improves the resolution much further and allows an optical resolution of $\sim 50 \mathrm{~nm}$ in the plane and $\sim 5 \mathrm{~nm}$ along the optical axis (perpendicular to the sample surface). This is achieved by a setup that uses a very small aperture through which light is transmitted. The aperture that is usually at the tip of an optical fiber is brought to almost-contact distance from the object and the reflected light is measured. The sample is than scanned point by point to reconstruct the reflection image (it can also be fluorescence or scattering). In such a way, even though the light that comes out of the tip is totally diffracted, the very short distance from the tip to the sample ensures that the signal mainly results from the surface that is next to the tip. This method therefore bypasses the diffraction limit by measuring at distances that are small enough so that the diffraction is still negligible. Nevertheless, near-field microscopy is limited to a thin layer of the sample surface $(\sim 10-20 \mathrm{~nm})$.

Other methods that improve the optical resolution include multi-photon microscopy ${ }^{5}$ and $4 \mathrm{Pi}$ microscopy ${ }^{6}$ but in practice, its improvement is similar to that of a confocal microscope. Another promising method is termed stimulated emission depletion (STED) ${ }^{7}$ and has been shown to achieve very high resolution of $33 \mathrm{~nm}(\lambda / 23)$. These methods are useful, have great potential and some of them are intensively used (e.g. confocal and multiphoton microscopy) but at least at this time these methods require sophisticated and expensive equipment.

In this work, we describe a new method that can be used for high resolution optical microscopy. It is based on a novel interaction of light with a metallic surface, when a periodic array of holes or grooves is patterned on the metallic surface.

\section{LIGHT TRANSMISSION THROUGH A METALLIC HOLE AND HOLE-ARRAY}

The theory of the transmission of light through a small hole (relative to the wavelength of light) in a metallic sheet was developed by Bethe in 1944. The main characteristics are that the intensity transmitted through the hole falls as $(\mathrm{d} / \boldsymbol{\lambda})^{4}$ $\left(10^{-3}\right.$ for a $100 \mathrm{~nm}$ hole $)$ and the light is totally diffracted, i.e. after passing through the hole, the light is spread to an angle of $2 \pi$ Steradians.

Lately, it has been shown that apertures that are smaller than the wavelength of light can transmit much more light than mentioned above, and even more striking, the light beam that passes the aperture has only a small diffraction. This is made possible by creating a periodic set of holes (or grooves) in a metallic sheet ${ }^{9}$. When manufacturing such a metallic hole-array (MHA) device, there are various degrees of freedom such as the type of metal and design of the periodic structure of holes. The characteristics of various structures have been studied ${ }^{10,11}$.

These effects probably occur due to the interaction of the light with the surface plasmons on both sides of the metallic sheet. Various theoretical models have been suggested with a satisfactory level of accuracy; see for example the work by L. Martin-Moreno ${ }^{12}$ and references therein.

MHAs form an interesting class of devices that can be used for various applications. A few applications that have been suggested include high-throughput near-field probes, high density data storage devices and lithography.

For a better understanding of the interaction of light with MHAs, it is important to understand the character of surface plasmons.

\section{PLASMONS AND SURFACE PLASMONS}

Plasmon describes the collaborative oscillation of electrons in a conductor (usually metal) and the electric field that results from it. When a sea of many free electrons oscillates, it creates an electric charge density. This charge density induces an electric field that affects the electrons' motion. At some frequencies (the plasmon frequencies), it can be shown that the oscillation of the electron sea can last for a relatively long time and span a relatively large region of the metal. The electric field that is associated with the oscillations of the electron sea is bound to the surface and is sometimes called an evanescent field.

In bulk material, the oscillation frequency can be found in a relatively simple way. Imagine a simple model of electrons oscillating in a metal cube and thereby forming a uniform surface charge density on the two opposite sides (see Figure 1). Assuming the volume density of the free electrons is $N$, the charge of an electron $-e$ and the displacement of the 
electrons relative to their equilibrium state is $x$, the surface charge density on each side will be $\sigma=N e x$ (with opposite signs on the two different sides). This will induce a uniform electric field inside the metal $E=4 \pi \sigma$ which will apply a force on each electron $F=-e E=-4 \pi N e^{2} x$. The equation of motion for each electron with mass $m$ will therefore be:

$$
m \ddot{x}=-4 \pi N e^{2} x
$$

which has the time-dependent solution:

$$
x=x_{0} e^{i \omega_{P} t}
$$

where $x_{0}$ is the oscillation amplitude and $\boldsymbol{\omega}_{\mathrm{p}}$ the plasmon frequency:

$$
\omega_{p}^{2}=\frac{4 \pi N e^{2}}{m} .
$$

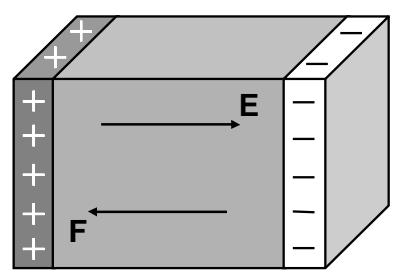

Figure 1 A simplified model of plasmon oscillations in metal. The electron displacement creates surface charge densities (with opposite signs). $\mathbf{E}$ is the electric field induced by the charge density and $\mathbf{F}$ is the force on an electron in the field.

Though simple, this solution provides understanding of the plasmons oscillations and describes the correct plasmon frequency of bulk plasmons in metal. This harmonic oscillation follows the rules of a quantized harmonic oscillator and therefore has quantized energy levels separated by $E=\hbar \omega_{p}$. When using the values of a typical metal, one finds that in silver $(\mathrm{Ag})$, the plasmon energy is approximately $12 \mathrm{eV}$ which is much larger than the energy of photons in the visible light range (1-3 eV). It can still be measured, usually by methods of electron loss spectroscopy.

A more complete solution of plasmon oscillations in bulk or on a surface requires to solve Maxwell's equations in matter (where the polarization vector plays a major role) together with the electron equation of motion (that provides a solution to the polarization as a function of the induced electric field). This set of equations can be solved simultaneously, for example see Feynman ${ }^{13}$ chapters 7 and 32.

The solution for surface plasmons can be found in a similar way, but is somewhat more complex because two sets of equations have to be written (in the metal and in the air) with the correct boundary conditions ${ }^{14}$. These solutions are best described by the dispersion curves. A dispersion curve results from the fact that there are actually many possible plasmon frequencies $\omega$ and each one of them relates to a different wave vector $k$ of the excitation. The dispersion curves of bulk and surface plasmons are shown in Figure 2A. They are given by the following equation in bulk ( $c$ is the speed of light):

$$
\omega=\omega_{p} \sqrt{\left(\frac{c k}{\omega_{p}}\right)^{2}+1}
$$

and for surface plasmons:

$$
\omega^{2}=\frac{\omega_{P}^{2}}{2} \cdot\left(1+2 \cdot\left(\frac{c k}{\omega_{p}}\right)^{2}-\sqrt{1+4 \cdot\left(\frac{c k}{\omega_{p}}\right)^{4}}\right) .
$$




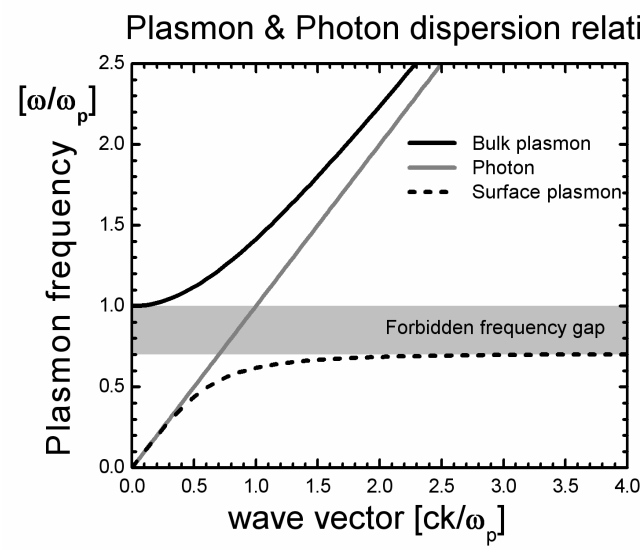

A

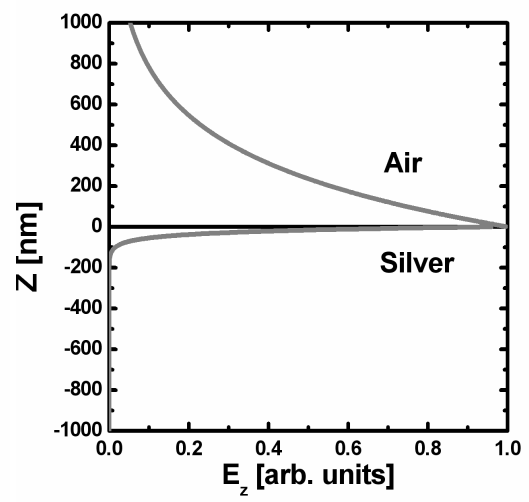

B

Figure 2 A: Dispersion curves for bulk and surface plasmons. The frequency and wave vector axes are normalized to the plasmon frequency. The photon dispersion curve is also shown. The fact that the photon and plasmon dispersion curves do not cross each other means that the interaction is not possible under normal conditions. B: Penetration depth of the plasmon electric field in the metal and air along the $Z$ axis (perpendicular to the surface) as calculated for Silver. The fields decay exponentially. Note the relatively large extent of the field in the air relative to metal.

The dispersion curve for surface plasmons shows the dependence on the dielectric functions of the metal and the other media (i.e. air):

$$
\begin{aligned}
& k_{z i}=\sqrt{\varepsilon_{i} \cdot\left(\frac{\omega}{c}\right)^{2}-k_{x}^{2}} \\
& k_{x}=\frac{\omega}{c} \cdot \sqrt{\frac{\varepsilon_{1} \cdot \varepsilon_{2}}{\varepsilon_{1}+\varepsilon_{2}}}
\end{aligned} .
$$

Here $k_{x}$ is the wave vectors of plasmons in the plane and $k_{z i}$ is the wave vector perpendicular to the surface. $\varepsilon_{i}$ is the dielectric function of the metal $(i=1)$ or the other media $(i=2)$ and $\omega$ is the plasmon frequency. Obviously both $k$ and the dielectric functions are complex. Especially for metal, the dielectric constant is usually complex with a negative imaginary part and a small positive real part.

The dispersion curves shown above can be used to find the exact electric field and charge density at any point in space. For surface plasmons a few points should be emphasized:

1. The plasmon amplitude exponentially decays when moving into the metal. It is therefore a surface phenomenon with a typical skin-depth of $\sim 20-50 \mathrm{~nm}$ (Fig. 2B). Therefore, a metallic sheet that is thinner than this is usually transparent, and it is opaque if it is more than twice as thick.

2. Above the metallic surface, the extent of the plasmon-induced electric field is much larger and can extend to a range of $\sim 500 \mathrm{~nm}$ (Fig. 2B). Also, the plasmon has a large extent in the plane and can be as large as 1 millimeter in some cases (depending on the metal and wavelength) ${ }^{14,15}$.

3. For large $k$, the surface plasmon frequency asymptotically reaches $\omega_{P} / \sqrt{2}$ which is sometimes defined as the surface plasmon frequency (Fig. 2A).

4. The fact that the photon and plasmon dispersion curves do not cross means that it is not possible to get a direct photon-plasmon interaction.

5. The plasmon frequencies are dependent also on the conductivity of the metal ${ }^{13}$ that adds a damping factor to the electron equation of motion Plasmon oscillation frequencies are also related to the screening effect in 
metals. Briefly speaking, the plasmon frequency is the cut-off frequency above which screening is negligible, but below which it is dominant.

Light can be still coupled to surface plasmons. The most common way is to use a prism to shine the light on the sample. Due to the high index of refraction of the prism (and large incident angles), the slope of the photon dispersion curve decreases and a crossing with the surface plasmon dispersion curve occurs ${ }^{14}$. Another method uses a periodic structure on the metal. In all these coupling methods, the electric field is bound to a shallow layer above the surface and is therefore called an evanescent field. This means that light that illuminates one side of the metallic sheet induces plasmons and an evanescent field on the other side of the metal, but light is not transmitted through the sheet.

In the last few years another interesting aspect of surface plasmons has been discovered when using a metallic sheet that has a periodic array of holes in it (or a periodic array of grooves with a single central hole). This is explained in the next section.

\section{METALLIC HOLE ARRAYS}

As mentioned in section 2, it has been recently shown that apertures that are smaller than the wavelength of light can transmit more light than the theoretical classical prediction ${ }^{8}$ and that the light beam that passes the aperture can have a very small diffraction. These phenomena occur when there is a periodic set of holes (or grooves) in a metallic sheet ${ }^{9,11}$.

The first effect in these structures is that the intensity of light that is transmitted through the hole is higher than the light that actually impinges on the hole (i.e. the transmission is larger than 100\%). This is due to the large interaction of light with surface plasmons when light falls on the metal in between the holes, and the transfer of this energy to the other side of the metal sheet. Few theories have been suggested to explain this phenomenon ${ }^{16,17}$. The second effect of MHA is the appearance of spectral peaks in the transmitted light. These peaks correspond to the crossing points of the photon and plasmon dispersion curves. To understand more about the transmission spectra, it is crucial to find the dispersion curves of a MHA. A third effect that has been found ${ }^{9}$ is the low diffraction of light that is transmitted through the hole (estimated to have an angle of $\sim 6^{0}$ ).

One model that can be used to find the surface plasmons in MHA assumes that the electron wave function on the surface has the same periodicity as the array. Therefore, the plasmon wave function will now have another periodic part that can be written as $e^{i\left(i G_{X}+j G_{Y}\right) \cdot \mathbf{r}}$ with $G_{X, Y}=2 \pi / a$, $a$ being the period length of the hole array (assuming a square-like array) and $i, j$ are integers. This periodicity results in an additional contribution to the plasmon wave vector and therefore allows the interaction with light. The solution to the plasmon energy levels can be found by using Equation 6 and including wave vector $\boldsymbol{G}$. The equation for the MHA solution is then written as:

$$
\left|\vec{k}_{S P}\right|=\frac{\omega}{c} \sqrt{\frac{\varepsilon_{1} \cdot \varepsilon_{2}}{\varepsilon_{1}+\varepsilon_{2}}}
$$

where the plasmon wave vector is:

$$
\vec{k}_{S P}=\vec{k}_{x, \text { photon }} \pm i G_{x} \pm j G_{y} \quad ; \quad i, j=1,2 \ldots \quad ; \quad G_{x, y}=2 \pi / a_{x, y} .
$$

Here, $\vec{k}_{x, \text { photon }}$ is the additional wave vector (momentum) that is added to the plasmon from the photon, and it is the component of the photon wave vector that is parallel to the surface. The dielectric functions are in general complex as mentioned above, especially for metal. One can see from equation 8 that the plasmon wave vector is made of two components; the photon wave vector in the surface plane and the wave vector contributed due to the plasmon periodic wave function as explained above. The addition of the periodicity has a dramatic effect on the dispersion curves and can be understood as folding the smooth-surface dispersion curve in parallel to the axes at values of $k_{x}=i G$. The resulting dispersion curves are shown in Figure 3. The plasmon energy levels (or wavelength on the right side) are shown as a 
function of the in-plane wave vector of the incident light $k_{x}$. These curves are calculated for a silver/quartz interface with a hole-array periodicity of $a=500 \mathrm{~nm}$. The numbers next to the curves indicates the quantum indexes $(i, j)$; some of the curves are degenerate. The fact that there are energy solutions for $k=0(1.61$ and $2.18 \mathrm{eV})$ means that even when the incident light is perpendicular to the surface, the interaction is still possible. When a MHA with a different distance between the holes is analyzed, the dispersion curves will shift; the energy lowers when the hole-distance grows, and vice versa.

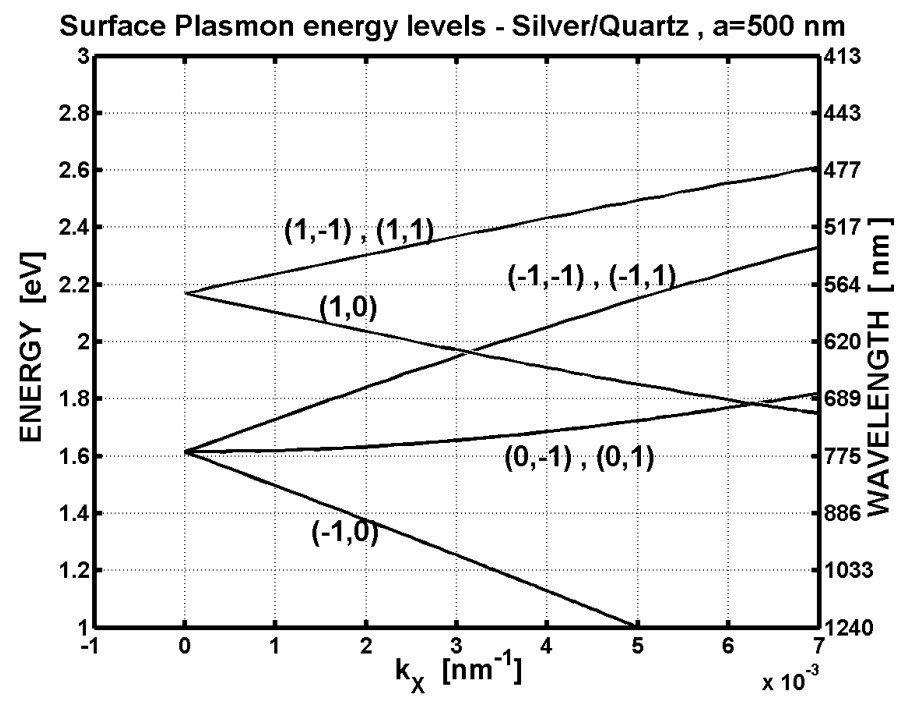

Figure 3 Dispersion curves for surface plasmons in a hole-array. These curves are calculated for silver/quartz with a hole-array with a periodicity of $\mathrm{a}=500 \mathrm{~nm}$. The numbers next to the curves indicate the quantum indices $(i, j)$; some of the curves are degenerate. Although not shown, the curves are symmetric around $\mathrm{k}=0$.

The small-diffracted beam mentioned above, was demonstrated in the case of a single hole ${ }^{9}$ surrounded by periodic grooves on both sides of the metal sheet. A full characterization of the phenomenon is still not known. It can be explained due to the interference of the surface plasmon field with the radiated light field that is transmitted through the holes. This effect may be used for high-resolution microscopy as we describe in the next section.

\section{MID-FIELD MICROSCOPE}

By combining a MHA with far-field collection optics we propose that a high resolution microscope that can measure three-dimensional objects can be constructed. The principle is shown in Figure 4 and we will consider a biological sample that is labelled with fluorescent probes. Collimated light illuminates the metal hole-array. The MHA is designed to have the appropriate transmission spectrum that matches the excitation spectrum of the fluorochromes that are used. The sample has to be brought to contact to the bottom side of the MHA while mounted to a high precision 3D stage. Assuming that the transmitted beams have a small dispersion, a small area of the sample will be illuminated with a diameter that is approximately the hole size. If there is fluorochrome in this area, it will fluoresce and the fluorescent signal will be collected with the far field optics (i.e. an objective lens) and relayed to a CCD. Each fluorescence spot will be imaged on the CCD with a diffraction pattern that is due to the fluorescence intensity and with a pattern that is the convolution of the spot size and the point spread function (PSF) of the collection optics. As long as the holes are farenough from each other (an order of $300 \mathrm{~nm}$ or more), there will be a very small overlap of adjacent PSF's and the data can be easily interpreted. To image the whole sample, it is necessary to scan the sample in three dimensions in steps that have approximately half of the hole size. Assuming a $50 \mathrm{~nm}$ hole, this is not difficult to do. There are technical difficulties that have to be overcome in order to achieve the type of measurement we describe, such as the requirement to scan the sample while it is still in contact with the hole-array. We believe that these challenges are doable. 
Because this microscope will have an illumination mechanism that is similar to a near-field microscope but collection optics that are far-field, we call this microscope a mid-field microscope.

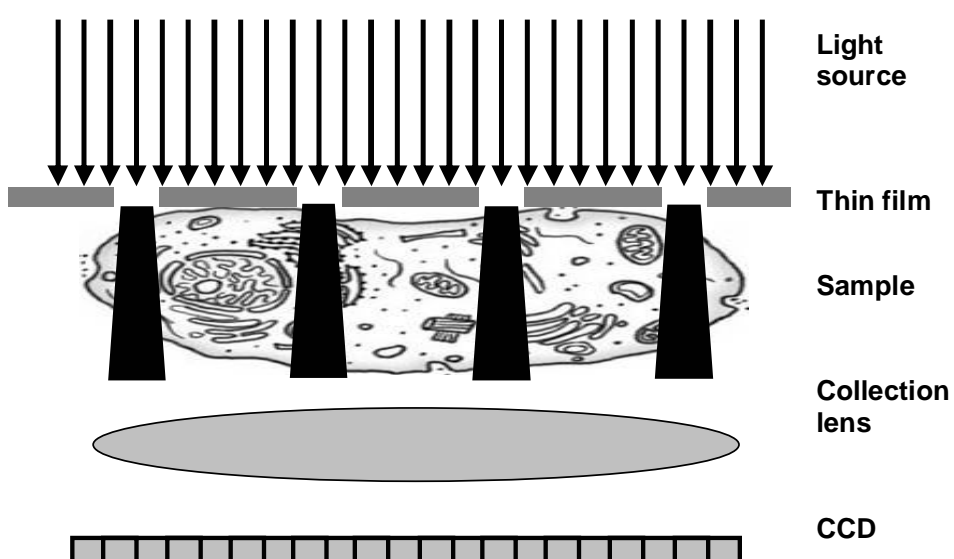

Figure 4 . Schematic description of the suggested mid-field microscope. Collimated light source impinge on a metallic hole-array and light beams with small diffraction exit at the bottom. These beams travel through the sample that is in contact with the metallic hole array. Fluorescent light is then collected with far-field optics to the CCD. Other optical elements that are required such as a filter in front of the CCD are not shown. For the measurement of the full threedimensions of the object, the sample has to be scanned.

\section{DISCUSSION AND SUMMARY}

The exact 3D resolution that will result is still unclear. Assuming that the technical challenges are overcome, this resolution may be better in the $X Y$ plane than along the optical axis. This is a result of the fact that if the illuminated light is not diffracted, a column of excitation light will pass through the sample. This will result in a fluorescence signal from a thick section of the sample and may therefore reduce the resolution along the optical axis. This problem and others will have to be examined.

It is also not clear what is the overall depth that can be scanned with high resolution. This is due to the fact that the light beam that comes out of the hole does have some diffraction, so that beyond a certain distance it will be larger than the far-field spot size $(\sim 200 \mathrm{~nm})$ and the advantage of the method will diminish. Further there will be the problem of scatter within the biological specimen confounding the beam diameter.

When compared to Near-field microscopy, the method has two advantages. One is speed; while a near-field microscope scans the sample with a single probe, the mid-field microscope we suggest here has many holes (say, 100x100) and therefore the measurement for a given area will be much faster. Another advantage will be due to the large depth-offield of the suggested mid-field microscope relative to approximately $20 \mathrm{~nm}$ in a near-field microscope. This may be significant especially for biological samples when a lot of the information is inside (e.g. a nucleus).

In summary, we have described a new type of microscope that we call a mid-field microscope. It is based on a metal hole-array device with holes that have sub-wavelength dimensions in combination with a precise 3D stage and far-field collection optics. The method is based on the photon-plasmon interaction of light with a thin metallic hole-array that is still not fully understood. Even though the method is challenging, it has both potential applications in microscopy and physical insight that has yet to be explored. We are in the process of performing preliminary measurements with the first set of hole-arrays that we have manufactured. 


\section{ACKNOWLEDGEMENTS}

We would like to thank Dr. Victor Goldman for the valuable and insightful discussions.

This work was partially supported by the Physics for Technology programme of the Foundation for Fundamental

Research in Matter (FOM), the Delft Inter-Faculty Research Center Intelligent Molecular Diagnostic Systems (DIOC-

IMDS) and the Delft Inter-Faculty Research Center LifeTech (DIO-LifeTech).

\section{REFERENCES}

1. E. Abbe, "Beitrage zur Theorie des Mikroskops und der Mikroskop ischen Wahrnehmung". Archiv fur Microskopische Anatomie, 9, 413-420, 1873.

2. M. Born and E. Wolf, Principles of Optics, Cambridge University Press, Cambridge, ed. 7, 1999, chapter 8.

3. T. Wilson and C. Sheppard, Theory and practice of scanning optical microscopy, London: Academic Press, 1984.

4. E. Betzig, J.K. Trautman, T.D. Harris, J.S. Weiner, and R.L. Kostelak, "Breaking the diffraction barrier: optical microscopy on a nanometric scale", Science, 251, 1468-1470, 1991.

5. W. Denk, J.H. Strickler and W.W. Webb, "Two-photon laser scanning fluorescence microscopy", Science 248, 7376, 1990.

6. H. Kano, S. Jakobs, M. Nagorni and S.W. Hell, "Dual-color 4Pi-confocal microscopy with 3D-resolution in the 100 nm range", Ultramicroscopy 90, 207-13, 2001.

7. S.W. Hell, "Toward fluorescence nanoscopy", Nature Biotechnology 21, 1347-1355, 2003.

8. H.A. Bethe, "Theory of diffraction by small holes", Physical Review 66, 163-182, 1944.

9. H.J. Lezec, A. Degiron, E. Devaux, R.A. Linke, L. Martin-Moreno, F.J. Garcia-Vidal and T.W. Ebbesen, "Beaming Light from a Subwavelength Aperture", Science 297, 820-822, 2002.

10. H.F. Ghaemi, T. Thio, D.E. Grupp, T.W. Ebbesen and H.J. Lezec, "Surface plasmons enhance optical transmission through subwavelength holes", Physical Review B 58, 6779-6782, 1998.

11. D.E. Grupp, H.J. Lezec, K.M. Pellerin, T.W. Ebbesen and T. Thio, "Crucial role of metal surface in enhanced transmission through subwavelength apertures", Applied Physics Letters 77, 1569-1571, 2000.

12. L. Martin-Moreno, F.J. Garcia-Vidal, H.J. Lezec, A. Degiron and T.W. Ebbesen, "Theory of highly directional emission from a single subwavelength aperture surrounded by surface corrugations", Physical Review Letters 90, 167401/1-4, 2003.

13. R.P. Feynman, R.B. Leighton and M. Sands, The Feynman lectures on physics, Addison-Wesley Publishing Company, California, ed. 6, 1964.

14. H. Raether, Surface Plasmons on smooth and rough surfaces and on gratings. G. Hohler, E.A. Niekisch, Eds., Springer Tracts in Modern Physics vol. 111, Springer-Verlag, Berlin, 1988.

15. W.L. Barnes, A. Dereux and T.W. Ebbesen, "Surface plasmon subwavelength optics", Nature 424, 824-830, 2003.

16. J.A. Porto, F.J. Garcia-Vidal and J.B. Pendry, "Transmission Resonances on Metallic Gratings with very narrow slits", Physical Review Letters 83, 2845-2848, 1999.

17. L. Martin-Moreno, F.J. Garcia-Vidal, H.J. Lezec, K.M. Pellerin, T. Thio, J.B. Pendry and T. W. Ebbesen, "Theory of Extraordinary Optical Transmission through Subwavelength Hole Arrays", Physical Review Letters 86, 11141117, 2001.

* Y.Garini@tnw.tudelft.nl; Tel: +31-15-278-8574, fax: +31-15-278-6740, www.ph.tn.tudelft.nl/ yuval/index.htm. 